\title{
分析《碧奴》中的孟姜女的性格及其与当时女性看待丈夫的对比
}

\section{ANALYSIS OF THE CHARACTER OF BI NU "MENGJIANGNU" BY CONTRASTING WOMEN'S VIEW ON HUSBAND THROUGH AGES}

\author{
Wiliam Wijaya \\ Chinese Department, Faculty of Humanities \\ Jln. Kemanggisan Ilir III No.45, Kemanggisan-Palmerah, Jakarta Barat 11480 \\ wiliam_wijaya777@yahoo.com
}

\begin{abstract}
Novel BINU is one of Sutong's excellent works. Through Binu, the character which is called Meng jiangnu, could see weak women in order to find her lovely husband exhausted all its effort to walk a long road. Meng jiangnu's story is one of the four chinese's ancient legendary loves. Other are Niu Lang Zhinu, Liang Sanbo Yu Zhu Ying Tai, and Bai She Zhuan, have been widely spread for thousands of years. The legend of Meng jiangnu has been spread with oral tradition in folkloric. The earliest legend can be traced back to the "Zuo Zhuan". Through Novel BINU, the author wants to observe "Examining Meng jiangnu characterization by contrasting ways of women's view through ages in Novel BINU by Sutong". And then, the author also introduces Novel BINU, Meng jiangnu myths, and through XINGGE, to examine the characterization of Meng jiangnu. By examining the character Meng jiangnu, the author finds that Meng jiangnu had melancholy and choleric character. Both characters are shown out in difficulties. Meng jiangnu treated her husband better than women in that age. The women in that age just cried in waiting the fate changes.
\end{abstract}

Keywords: Bi Nu, Meng jiangnu, personality, view on husband

\section{内容提要}

《碧奴》是苏童一本优秀的小说作品。透过这本书里边的人物碧奴也常被称为孟姜女, 可以看到一 个柔弱的女子为了寻找她心爱的丈夫用尽全力走了很长的路途, 从一个柔弱的女子变成一位坚强有智慧 的女子。孟姜女故事是中国古代四大爱情传奇之一，其他三个是《牛郎织女》、《梁山伯与祝英台》和 《白蛇传》, 千百年来一直广为流传。孟姜女的传说一直以口头传承的方式在民间广为流传, 最早的传 说可上溯到《左传》。笔者透过苏童的小说故事《碧奴》想要研究孟姜女的性格及其与当时女性看待丈 夫的对比。除了那之外, 笔者还会简介《碧奴》这本小说, 简介孟姜女的神话故事与透过《性格》来分 析孟姜女的性格。笔者从这些分析得知孟姜女具有完美型与力量型性格, 这两种性格类型在困难中会展 现而出。然后, 孟姜女在于看待丈夫胜过当时女性看待丈夫, 孟姜女把丈夫看的比她性命还要重要, 而 当时女性只会哭哭啼啼等待着命运的转变。

关键词：《碧奴》, 孟姜女, 性格, 看待丈夫 


\section{前言}

笔者通过《碧奴》苏童的作品之一, 想要研究孟姜女这么一位常常在历史上被忽略的神话 人物的性格及其与当时女性看待丈夫的对比。笔者能够选择《碧奴》这本小说, 是因为一方 面是很欣赏苏童的作品, 另一方面是被小说里边的人物孟姜女给吸引住了, 所以想更加深地 研究孟姜女与透过这个研究, 笔者想让更多人知道是什么原因能让这神话般的人物穿越时空 一直口传到今日。除了这意外, 笔者还会简介《碧奴》这本小说, 简介孟姜女的神话故事, 并 通过《性格》来分析孟姜女的性格。

\section{内容}

\section{《碧奴》}

《碧奴》是苏童的一个作品。 《碧奴》这本小说里边的角色碧奴也常被称呼为孟姜女, 有 着她特殊的角色演变。她与我们所读所知道的孟姜女神话故事有了好多变化。在苏童笔下，无 论是在故事或者碧奴这个角色都有着它各自的生命力, 像在言语表达, 苏童用了先锋派修辞 性的语言来强调当时的社会情景, 并在语言与现实之间拉开一种距离, 一种虚幻的神话式的 距离, 所以满有加分点。他有意削弱了历史苦难的现场感和实在性, 使它们具有想象和审议 的意味。生动的细节却具有触目惊心的真实效果, 以轻巧的语言讽刺和鞭挞着压迫的力 量。作品中碧奴的丈夫始终是缺席的, 但正是这种缺席, 使人物和作品更有一种飞翔的姿 态, 也使这种情感作了跨时代的认识和体味。正如作者所说: 一个家喻户晓的故事, 永远 是横在作者面前的一道难题, 每个人心中都有一个孟姜女。我对孟姜女的认识其实也是对 一个性别的认识, 对一颗纯朴的心的认识, 对一种久违的情感的认识。我对孟姜女命运的 认同其实是对苦难和生存的认识, 孟姜女的故事是传奇, 但也许不是一个底层女子的传 奇, 是属于一个阶级的传奇。

《碧奴》的故事本身是一个巨大的隐喻。在隐喻中讲述苦难和悲伤的故事, 可以说是一 部眼泪的传奇。在故事中有提起当时人们的哭泣是有着种种的规定与年龄和性别有关。哭 泣是留给那年龄小与还无法行走的孩子的, 所以为了能得到哭泣的权利, 那些在年龄上已 能行走的孩子愿意放弃行走的快乐, 而选择在地上爬行。但在残暴统治下, 这项权利却被 剥夺和扭曲了。为了想解脱自己想哭的愿望, 柴村使用祖宗所传下来的巫经, 这巫经能让 村里的女孩子从不哭泣, 也从不微笑, 即使再浓再深的哀伤都能用巫经来抵抗。除了柴村 以外, 还有一个村有特别的女儿经, 那就是桃村。桃村的女儿经具有奇特的排泪秘方, 有 的用耳朵哭泣, 嘴唇哭泣, 更神奇的用乳房哭泣。说到桃村就要说到碧奴, 碧奴使用头发 来哭泣, 让头发天天又酸又臭显得非常愚笨。别人家的女儿嫁的是商人, 地主, 再不好也 嫁个木工或铁匠, 只有碧奴嫁了孤儿岂梁, 得到的所有财产就是岂梁这个人, 还有九棵桑 树。《碧奴》的故事在苏童的笔下有着许多的感情色彩, 就像一个画家把一幅图画成有山 有水的画面。

\section{孟姜女神话故事}

孟姜女的故事，作为中国古代四大爱情传奇之一，其他三个是《牛郎织女》、《梁山伯与 祝英台》和《白蛇传》, 千百年来一直广为流传。孟姜女的传说一直以口头传承的方式在民间 广为流传。最早的传说可上溯到《左传》。另有, 同名的民歌、电视剧和越剧《孟姜女》。孟 
姜女这个神话故事一直流传到今日有了千百年的历史了。这个故事能流传到今天不仅是故事精 彩而已, 而是孟姜女这个人物有着一个特殊的优点, 那就是她各人有着对丈夫忠诚的心, 对每 个遇到困难的人她都难以袖手旁观的。当她知道他丈夫死了, 她依然对丈夫的爱毫无改变, 也 幸亏当时有她, 全部死去的苦役才都能得超度。就是因为她的这样的精神和善良, 让她死后, 人人都为纪念这个贵人, 就在山海关附近的一个山头上设立了一座小庙叫姜女庙。

孟姜女的神话故事说的是在很久以前, 有一户姓孟的人家, 在自己的院子里种了一棵瓜, 瓜秧随着院墙爬到隔壁的姜家结了瓜。当瓜成熟时, 就把它破开来! 竟然里边是一个又胖又 白，非常可爱的小娃娃。这两家高兴得不得了，给孩子取名为“孟姜女”，并一起把她抚养。时 间过得飞快，一转眼孟姜女就长成了一个漂亮的大姑娘。

这时恰巧是在封建时代, 在秦始皇的统治下, 好多男人无论他是书呆子, 渔夫或者是小贩 子, 只要是成年男人都被捉到北方为秦始皇修建那座巨大坚固的长城。在这个时候有一个书呆 子也正在逃避秦始皇兵马的追赶, 这位书呆子就逃进了孟家的花园, 想在那边躲个风头。他躲 在树后边, 躲着躲着, 但始终还是被孟姜女发现了。当孟姜女想要尖叫时, 这位书呆子叫孟姜 女高抬贵手不要再尖叫了, 因为看他不像是坏人就不再尖叫了, 孟姜女想要他老实地说出为什 么要在这里躲, 这书呆子说他叫范喜良, 是一位书生, 为了逃避秦始皇兵马的追赶才逃到这花 园里, 就这样把一切的真相全都告诉孟姜女。孟姜女详细地看范喜良不像是个坏人, 就立即去 向老员外报告。

老员外在后花园盘问范喜良的家乡住处, 叫什么名字, 为什么跳墙入院。范喜良一五一 十地作了口答。老员外见他挺老实, 知书达礼, 就答应把他暂时藏在家中。范喜良在孟家 躲藏的这些日子, 老两口见他一表人材, 举止大方, 就商量着招他为婿。跟女儿一商量, 女儿也同意。给范喜良一提, 范公子也乐意, 这门亲事就这样定了。

当天晚上, 范喜良与孟姜女就这样拜堂成亲, 摆了几桌酒席, 邀请亲戚好友来做热闹气 氛。俗话说: “人有旦夕祸福, 天有不测风云”, 快乐的期间也有着它各自的阶段。在他们拜 堂成亲不到几个小时, 一群衙役就大胆地间进来, 把孟姜女的丈夫范喜良捉去当苦役, 为 秦始皇建筑万里长城。看到丈夫被衙役捉走, 她大哭一场不知如何是好。

一天、一个礼拜、一个月、一年, 日子就这样飞快得过去了, 孟姜女盼望着丈夫的消 息, 更朌望着丈夫的归来, 但毫无线索。她就这样向父母告辞, 想去北方寻夫和为丈夫送 寒衣, 无论路途多么遥远, 自己多么辛苦, 她都坚持到底想去寻找那心爱的丈夫。到了北 方建筑长城的地方, 去问丈夫的情况, 得知丈夫因为过度劳累而病死, 而尸体被埋在长城 墙里边。当孟姜女得知丈夫已经死得这个消息, 就大哭一场, 也不知哭了多长时间, 直哭 得天昏地暗, 狂风怒吼。可能她的哭声感动了天, 把那八百里长的长城都哭倒了。那些被 埋在墙内的劳动者的户骨, 全都露了出来。但因尸骨太多了, 她无法分辨出丈夫的尸骨, 她就把手指头咬了, 把血滴在骨头上才认出来那是她丈夫的尸骨。当她想把丈夫尸骨带走 时, 秦始皇与兵士来到长城倒塌之地, 想看谁有那么大的本领能把那八百里的长城哭倒了。 当秦始皇看到孟姜女时就被她的美貌给吸引住了, 不但不处置她反而想把她封为正宫娘娘。起 初孟姜女想要直接地拒绝他, 但她突然想起一好办法! 聪明的孟姜女说: “除非你答应我三件 事! 第一, 设立灵堂, 为我的丈夫以及所有死去的苦役超度亡灵; 第二, 好好安葬我的丈夫范 喜良; 第三, 我要游海三天。”

秦始皇开始有些犹豫, 可是一看见漂亮的孟姜女, 最后还是妥协了。于是下令设灵 堂, 召来僧人为死去的人超度, 并好好安葬了范喜良。孟姜女看见自己的愿望已经达到 
了, 就来到海边走到船边上, 纵身一跃, 跳进渤海自尽了。孟姜女跳进渤海的消息很快传 到了各地。人人为了表达对她的大恩大德就在山海关附近为她设立一座小庙叫“姜女庙”。

\section{气质定义}

每个人在这世间都有着各自独特的气质，一人与另一人都具有各自不同的优点与缺点。气 质是人的个性心理特征之一, 它是指人的认识、情感、言语、行动中, 心理活动发生时力 量的强弱、变化的快慢和均衡程度等稳定的动力特征。主要表现在情绪体验的快慢、强 弱、表现的隐显以及动作的灵敏或迟钝方面, 因而它对人的全部心理活动表现染上了一层 浓厚的色彩。它与日常生活中人们所说的“脾气”、“性格”、“性情”等含义相近。

透过通俗来解释, 气质在现在的社会所表现的是一个人从内到外的一种内在的人格魅 力, 然后所发挥的一个人内在鬼力的质量的升华。所指的人格魅力有很多的, 比如修养品 德、举止行为、待人接物、说话的感觉等, 所表现的有高雅: 高洁、恬静、温文尔雅、豪 放大气不拘小节立竿见影等。所以, 气质并不是自己所说出来的, 而且自己长久的内在修 养平衡以及文化修养的一种结合，是持之以恒的结果。

气质是一个古老的心理学问题。早在公元前 5 世纪, 古希腊著名医生希波克拉 (Hippocrates) 就特提出了 4 种体液的气质学说。他认为人体内有四种体液: 血液（来自 拉丁语一sanguis）、粘液（来自希腊语一phlegma）、黄胆汁（来自希腊语一chole）和黑 胆汁（来自希腊语一melanoschole）。四种体液谐调, 人就健康, 四种体液失调, 人就会生 病。希波克拉特曾根据哪一种体液在人体内占优势把气质分为四种基本类型：多血质 (Sanguine)、胆汁质 (Choleric) 、粘液质 (Phlegmatic) 和抑郁质(Melancholy)。多血质的 人体液混合比例中血液占优势, 胆汁质的人体内黄胆汁占优势, 粘液质的人体内粘液占优 势, 抑郁质的人体内黑胆汁占优势。几个世纪以后, 罗马医生哈林 (Galen) 用拉丁语 “emperametnum”一词来表示这个概念。这就是“气质”（temperament）概念的来源。

在 1992 年有一位 美国女士叫弗洛伦斯.妮蒂雅(Florence Littauer)把自己对性格的看法 以及详细分析都把它记载在《性格》书上。《性格》里边把人的性格分为四种基本性格类 型, 那就是活泼型 (sanguine)、完美型 (melancholy)、力量型（choleric）与和平型 (phlegmatic) 。在这本书里边不用多血质、抑郁质、胆汁质和粘液质等这四种气质, 而用 的是活泼型(多血质)、完美型(抑郁质)、力量型（胆汁质）与和平型（粘液质）。笔者用四 性格类型而不用四个气质类型的说法，因为这四性格类型的说法比较著名也更容易记住。 活泼型的人活泼和爱与别人交流。完美型的人喜爱有自己的空间, 不喜爱和别人说话, 但这个 类型的人容易对别人付出感情或者说容易感动。力量型的人很严肃, 做事挺认真。和平型的人 做事冷静，经过思考才做决定。这些只有对性格类型的简单分析而已。

\section{性格类型}

\section{活泼型}

活泼型的人很容易就能认出来的。他们做某种事时都应人注目, 因为他们喜欢在别人面前 表现他们自己。除了那以外, 他们也是一个活泼、开朗、爱说话、喜欢与别人交流、也是舞台 上的人才。这种类型的人缺点是说话常常不经过思考就说出来, 但他们并没恶意, 脾气暴躁缺 乏毅力。活泼型的人看上去似乎在任何情况下多很开心, 但实际上, 他们非常渴望得到别人的 关注, 接受和认可。他们需要被爱和被别人接受, 他们需要听众和掌声。批评和贬低会使他们 消沉。 


\section{完美型}

完美型的人的座右铭是“如果值得做的，就要做得好”。这与他们做得多快无关，而与他们 做得多好有关。质量总是比数量重要。如果一件工作有完美型的人负责, 便会知道这件工作会 做得很好而且能够按时完成。这类性的人最有天赋、最有创造力, 他们可能有音乐、哲学、文 学等多方面才华。他们喜爱孤独和不善与别人交流。虽然如此, 他们在感情方面非常丰富、感 情真诚、易受感动、甚至敢于为他人牺牲。他们是一位爱听别人困难的好听众, 也能为遇到困 难的朋友想出解决问题的办法。他们不会满足于只看到事物的外表, 而他们想要深刻地研究事 物的内涵。这类型的人的缺点是容易悲观, 想一件事常往坏的方向去想, 他们容易怀疑别人对 他们的表扬和他们做事只强调前后次序和组织。

\section{力量型}

力量型的人永远充满着动力, 他们会充满理想, 他们勇于攀登高不可攀的顶峰, 就是因 为那勇敢的精神、意志坚决、容易做出决定和天生有着领袖导致他们适合当一位领导。力量型 的人是自发性的，他们必须改变任何他们看来不合适的东西，并纠正任何加诸于那些无助的人 身上的错误。力量型的人会为信念挺身而出, 高攀真理。这类型的人没达到目标, 就继续克服 阻碍, 继续前进。力量型的人在生活中有一个难于扮演的角色。他们心中有答案, 他们知道做 什么, 能快速做出决定, 会帮助他人, 但是他们很少会受到欢迎, 因为他们的自信和主张使别 人感到不安。他们的领导能力往往令他们看来只懂指使别人。这类型的人缺点是顽固、好争吵 和无同情心。受别人的重视与能拥有控制权是这个类型的人基本的要求。

\section{和平型}

和平型的人最容易与别人相处。他们无论在哪儿都是受人喜爱。这类型的人无论有朋友 没朋友都会自得其乐。几乎没有什么可令他们烦恼, 而他们喜欢旁观过客。这类型的人能有好 多朋友的原因之一是他们是很好的听众。在队中，他们愿意听而不说话。无论环境如何的变 化, 他们都保持安静。他们这样做是为了要求和平与避免冲突。这类型的人天生就有良好的人 际关系。他们性格随然闲适、平静、心智平静、有耐心, 和平、不侵犯他人并且心情愉快。这 类型的缺点是胆小、平淡、犹豫不觉、缺少热情和冷漠。他们基本的要求是受到他人的珍惜与 尊重。在下边，笔者会设下四个类型的表格那就是：活泼型、完美型、力量型与完美性。

\section{性格分析表}

\section{活泼型性格分析}

\begin{tabular}{|c|c|c|c|}
\hline \multicolumn{4}{|c|}{ 活泼型性格 } \\
\hline \multicolumn{3}{|c|}{ 优点 } & \multirow{2}{*}{ 缺点 } \\
\hline 情感方面 & 对待工作 & 作为朋友 & \\
\hline 予人好感 & 工作主动 & 容易交朋友 & 散漫 \\
\hline 健谈 & 找寻新事物 & 热爱别人 & 健忘 \\
\hline 聚会的灵魂 & 注重表面 & 喜欢赞扬 & 好插嘴 \\
\hline 幽默感 & 富有创造性 & 看似兴奋 & 难预测 \\
\hline 美好的回忆 & 充满干劲 & 令人羡慕 & 即兴 \\
\hline
\end{tabular}




\begin{tabular}{|c|c|c|c|}
\hline 自如地控制听众 & 闪电式开始 & 不怀恨 & 放任 \\
\hline 情绪化及感情外露 & 鼓励他人参与 & 很快道歉 & 易怒 \\
\hline 好表现 & & 避免沉闷 & 天真 \\
\hline 好奇 & & 喜欢即兴的活动 & 喜获认同 \\
\hline 舞台上的人才 & & & 喋喋不休 \\
\hline 天真无邪 & & & 生活紊乱 \\
\hline 现实 & & & 大嗓门 \\
\hline 性情善变 & & & 不专注 \\
\hline 诚挚 & & & 烦操 \\
\hline 孩子气 & & & 善变 \\
\hline
\end{tabular}

\section{完美型性格分析}

\begin{tabular}{|c|c|c|c|}
\hline \multicolumn{4}{|c|}{ 完美型性格 } \\
\hline \multicolumn{3}{|c|}{ 优点 } & \multirow{2}{*}{ 缺点 } \\
\hline 情感方面 & 对待工作 & 作为朋友 & \\
\hline 深思熟虑 & 预先作计划 & 交友谨慎 & 不宽怒 \\
\hline 善于分析 & 完美主义 & 甘愿留在幕后 & 怨恨 \\
\hline 严肃 & 高标准 & 避免引起注意 & 挑剔 \\
\hline 有目标 & 注重细节 & 忠诚可靠 & 无安全感 \\
\hline 有天分 & 善始善终 & 会聆听抱怨 & 不受欢迎 \\
\hline 富有创造能力 & 有条理有组织 & 解决别人问题 & 难于取悦 \\
\hline 富音乐艺术胞 & 整洁（整齐清洁） & 很关心他人 & 悲观 \\
\hline 冷静富有诗意 & 讲求经济效益 & 情感丰富 & 孤芳自赏 \\
\hline $\begin{array}{l}\text { 追求完美 } \\
\text { 对他人 }\end{array}$ & $\begin{array}{c}\text { 善于发现问题 } \\
\text { 有创造性 }\end{array}$ & 易受感动 & 消极 \\
\hline 反映敏感 & 解决方法 & 寻求理想伴侣 & 不善交际 \\
\hline 自我牺牲 & 勤俭节约 & & 过分敏感 \\
\hline 有责任心 & 善用图标, 数据目录 & & 抑郁 \\
\hline \multirow[t]{6}{*}{ 理想主义 } & 分析问题 & & 内向 \\
\hline & & & 情绪化 \\
\hline & & & 有戒心,多疑 \\
\hline & & & 孤僻 \\
\hline & & & 好批评 \\
\hline & & & 报复性 \\
\hline
\end{tabular}




\section{力量型性格分析}

\begin{tabular}{|c|c|c|c|}
\hline \multicolumn{4}{|c|}{ 力量型性格 } \\
\hline \multicolumn{3}{|c|}{ 优点 } & \multirow{2}{*}{ 缺点 } \\
\hline 情感方面 & 对待工作 & 作为朋友 & \\
\hline 天生领导者 & 目标主导 & 不太需要朋友 & 专横 \\
\hline 活力充沛及主动 & 纵观全局 & 为团体而工作 & 无同情心 \\
\hline 急迫需要改变 & $\begin{array}{c}\text { 善于管理 } \\
\text { 寻求实际的解决 }\end{array}$ & 会领导及组织 & 逆反 \\
\hline 不容有错 & 方法 & 总是正确 & 急躁 \\
\hline 意志坚决, 果断 & 委派工作 & 善于应变 & 固执 \\
\hline 非情绪化 & 坚持生产 & & 自负 \\
\hline 自立自足 & 设定目标 & & 好争吵 \\
\hline 充满自信 & 促成行动 & & 工作狂 \\
\hline \multirow[t]{2}{*}{ 能运作一切 } & 越挫越奋 & & 不容忍 \\
\hline & & & 狡猾 \\
\hline
\end{tabular}

\section{和平型性格分析}

\begin{tabular}{|c|c|c|c|}
\hline \multicolumn{4}{|c|}{ 和平型性格 } \\
\hline \multicolumn{3}{|c|}{ 优点 } & \multirow{2}{*}{ 缺点 } \\
\hline 情感方面 & 对待工作 & 作为朋友 & \\
\hline 性格低调 & 熟悉可靠 & 容易相处 & 缺芝热情 \\
\hline 易相处, 轻松 & 平和无异议 & 开心愉快 & 胆小 \\
\hline 平静，镇静 & 由行政能力 & 无攻击性 & 犹豫不决 \\
\hline 耐心，易适应 & 调解问题 & 好的聆听者 & 平淡 \\
\hline 一成不变的生活 & 避免冲突 & 尖刻的幽默 & 无目标 \\
\hline 仁慈善良 & $\begin{array}{c}\text { 善于面对压力 } \\
\text { 寻求容易的 }\end{array}$ & 喜爱旁观 & 冷漠 \\
\hline 隐藏内心的情绪 & 解决方法 & 有很多朋友 & 担忧 \\
\hline \multirow[t]{7}{*}{ 乐天知命 } & & 同情与关心 & 平淡 \\
\hline & & & 无异议 \\
\hline & & & 喃喃自语 \\
\hline & & & 缓慢 \\
\hline & & & 懒惰 \\
\hline & & & 勉强 \\
\hline & & & 妥协 \\
\hline
\end{tabular}




\section{孟姜女性格分析}

通过《性格》来列出的四个类型的优点与缺点和利用《碧奴》中的故事演变和对话, 笔者 想更加深地分析孟姜女到底是属于哪一个类型的性格。

孟姜女的性格是完美型, 她也具有力量型的一些特点:

一、孟姜女具有完美型性格的特征:

（一）消极（反面的）

好多人情不自禁地叫了起来: “你这死脑筋的女子, 别再往城外跑了, 左手有了. 右手也 有了，再跑就不盖那徽印了，当场杀头啦！”（蓝袍文章 178 页）

\section{（二）挑剔}

有人好心地邀请她到窝棚里过夜，她看见棚里有几个半大的少年，怎么也不进去。好心人 给他气坏了, 说: “也不看看自己过的什么日子, 还这么臭讲究! ” (蓝袍文章 178 页)

那卖衣服的夫人看到碧奴一直不停地捏她卖的东西, 就拿一个树枝做的衣叉往碧奴的手上 叉, 然后她对碧奴说: “你还怨我叉你的手呢, 你在这里站了半天了, 从鞋子捏到帽子, 从帽 子捏到冬袍，你什么都不买，棉花捏出个厚薄来，又有什么用！”（蓝袍文章 179 页）

\section{（三）＼cjkstart不受欢迎}

那守摊的是一个精明泼辣的夫人，拿了一个树枝做的衣叉，谁摸她的衣服就用衣叉叉谁的 手。碧奴的手被叉了好几次, 她说: “大姐, 你这样凶, 我又不偷你的衣服, 我就是捏捏里面 的棉花, 看棉花够不够厚。”（蓝袍文章 178 页)

\section{（四） 勤俭节约（俭省）}

五谷城人人都知道的省钱道嘛, 富人家的丧事一办完, 就有一帮穷人等在门外捡丧袍, 捡回去染了颜色, 当新衣服穿! 这一番话提醒了碧奴, 她一边留恋地抚摸那袍子, 眼睛亮了起 来: “老伯，你知道五谷城里哪儿有染铺的？”（蓝袍文章 180 页）

\section{（五） 善于分析}

碧奴守护着蓝袍, 那是她漫长的北上旅途中得到唯一的一件财产, 她端详着这件来之不易 的财产，忍不住地伸手去摸它，摸了几次她有开始发愁了： “毕竟是一件丧袍, 虽然看上去是 新的, 袍子的样式还是丧袍的式样, 她要是把它带到沽衣街去, 那些精明的旧衣贩子也许一眼 就看出来了, 就算他们看不出来, 这么一件染过颜色的旧袍子, 怎么换的到岂梁的冬衣? ” (蓝袍文章 182 页)

从孟姜女完美型性格分析得知：孟姜女是一位固执、挑剔、节省和善于分析的人。在别人 劝她不要再上前时, 她任然反面地向前走。在别人邀请她到窝棚里过夜, 她挑剔地拒绝那个 人。在卖衣服的地方, 她的手给卖衣服的人用衣叉叉了好几次显得非常不受欢迎。当孟姜女 得知好多五谷城的穷人专门捡富人穿过的丧袍, 然后带回去拿来重新染色当作新衣服穿, 就向 一个老伯问染铺在哪里, 为了想要把自己的丧衣染色。她想把丧袍卖给旧衣贩子, 但怕他们不 要接受。如果他们接受, 这个丧袍哪里能换到岂梁的冬衣, 孟姜女一直把事情慢慢地分析。 
二、孟姜女也具有力量型的特征:

(一) 固执

碧奴坚强地抱着那件丧袍, 追着一个老染工跑。老染工说: “你这女子怎么这样强（固 执）呢? 也不看看这染房是不是你来的地方, 你没有见我们忙着给袁将军家小姐染嫁妆呢, 哪 有闲心染你这件烂丧袍？”（蓝袍文章 180 页）

有人被碧奴的哭动了恻隐之心, 上去拉扯碧奴: “哭不得, 哭不得, 进城门不能哭的, 这 是五谷城一百年的老规矩！” 碧奴甩开了所有的手, 固执地坐在地上, 坐在地上哭, 哭得泪水 四溅。”（五谷城文章 166 页）

（二）不容忍

碧奴气得脸都白了, 站在磨盘上对高台上的小女孩说：“身上臭就该让狗欺负吗？谁愿意 做流民？谁愿意守着势利眼的五谷城？都是没办法呀，离家好几百里了, 出来容易回去难！”

(蓝袍文章 182 页)

从孟姜女力量型性格分析得知: 孟姜女是一位固执和不容忍的人。当孟姜女想要把丧袍染 时, 就追着一个老染工固执地要他把她的丧染色。有一人劝孟姜女不要在五谷城哭泣, 但她 不听那个人的劝导固执地在地上哭泣。一个小女孩把狗放出来咬孟姜女, 孟姜女气得脸都白忍 不住骂了那小女孩几句。

七、孟姜女的性格及其与当时女性看待丈夫的对比

在这儿笔者会把孟姜女的性格及其与当时女性看待丈夫的对比, 各自地分析为了想得知她 们是如何看待丈夫的。

一、孟姜女看待丈夫

岂梁失踪的那天中午, 碧奴还只会用头发哭泣。她站路上眺望北方, 发䯽上的泪, 雨点般 地落下来，打湿了青色罗裙。（哭泣文章 11-12 页）

碧奴站在自己的泪光里, 正在收拾一只巨大的包裹, 包裹里有一套手工精美镶有五彩大纹 的冬袍, 还有腰带, 还有兔皮鞋。她们都猜到那是给岂梁的包裹, 谁不想给㫚忙离家的男人准 备一只大包裹呢? 她们问碧奴那么好的冬袍要花多少钱, 碧奴说不上来是多少钱, 她是用桑园 里九棵树加上三匾茧丝跟织房换的。女人们惊叫起来说: “碧奴你把桑树三匾茧丝换了, 以后 怎么过日子? ” 碧奴说: “岂梁不在, 这日子过也罢, 不过也罢。” 女人们又问碧奴: “你准 备了这么好的包裹，让谁捎到大燕岭去呢？”碧奴说：“没有人捎去，我自己送过去。”（哭 泣文章 14 页)

农妇们注意到她的手已经血肉模糊, 手过留痕, 草垛上留下了一串红色的血星星, 他们 说: “没见过你这么痴情女子呀! 我们十三里铺的男人也都上了大燕岭, 这么近的路, 也没有 人像你一样寻夫的, 你家男人就是个下凡的神仙, 也犯不上这样爬, 看看你的手, 你的膝盖, 你自己在流血呀! 你偏偏还要带着这石头, 爬到大燕岭就怕石头还在, 你人不在了! (十三里 铺文章 229 页)

\section{二、当时女性看待丈夫}

她看见商英的妻子祁娘和树的妻子锦衣也站在路上, 面向北方, 紧紧地咬着牙齿, 她们的 丈夫也失踪了。祁娘用她的耳朵哭, 她的耳朵在阳光下发出了一片泪光, 而锦衣仍然在用少女 
的秘法哭泣, 由于她不久前产下了一个婴儿, 正在哺乳期, 她的泪水混杂着乳汁流下来, 罗裙 尽湿，人就像从沟里爬上来的。（哭泣文章 12 页）

有一个绿衣女子向碧奴请教, 她该如何做才能让丈夫在大燕岭不冻死, 碧奴就说: “你回 去呀! 回家把你丈夫最暖的冬衣找出来, 明天趁着太阳好, 放到太阳地里晒一晒, 晒好了就可 以送到大燕岭去啦! ”但绿衣女子对她说: “我走不了那么远的路, 我身子骨弱, 我走去一定 会死在路上的。”（人市文章 44-45 页）

这些来自青云郡的挑夫追着山路上的水迹疾步如飞, 很轻易地追到了碧奴。可是看见碧奴 的泪脸, 他们就摇摇头走了, 失望地说: “不是我媳妇, 我就知道我媳妇吃不了那个苦, 不会 是我的媳妇！”（长城文章 241 页）

从孟姜女的性格及其与当时女性看待丈夫的对比得知：孟姜女是一位痴情的人，她愿意把 桑园里九棵树加上三㻞茧丝和织房跟一套手工精美镶有无彩大纹的冬袍换, 而把这套冬袍亲自 地送到大燕岭那么远的地方。在十三里铺要去大燕岭的路途, 孟姜女愿意背着石头在地上爬 行, 为了想让山神不要找她丈夫麻烦。当时女性看待丈夫, 只会哭哭啼啼看待丈夫离去, 贪 生怕死等待着命运的演变。

\section{结论}

通过《碧奴》中的孟姜女加上四种类型性格的理论分析得知, 碧奴或常被称呼为孟姜女具 有了两种类型性格那就是完美型和力量型。在于遇到苦难时, 她的完美型的善于分析和固执与 不容忍的力量型性格会展现而出。然后, 孟姜女与当时女性用不同的方式对待丈夫的对比。孟 姜女看待丈夫比她自己的性命更加重要, 虽然她知道去大燕岭的路途很长, 但她不怕吃苦地用 尽力为心爱的丈夫送冬袍。当时女性看待丈夫只是哭哭啼啼没有动静，没去看丈夫，只是把一 切都交给命运来做主。

\section{参考文献}

[1] 倪小龙·孟姜女 $[M] \cdot$ 江苏: 江苏古箱出版社， 2000 .

[2] 任溶溶·绘体中国故事（中国民间故事（上））[M]·杭州:浙江少年儿童出版社,2008.

[3] 韩鉴堂·中国文化[M]·北京:北京语言大学出版社,2005.

[4] 蒋鸿雁、陈烔 孟姜女[M] 深圳: 海天出版社, 2005.

[5] 苏童·碧奴-关于孟姜女传说[M]·重庆:重庆出版社，2006.

[6](美)弗洛伦斯·妮蒂雅·性格解析[M]·北京：经济日报出版社，1998. 
[7](美)弗洛伦斯·妮蒂雅·性格[M]·北京：经济日报出版社，2001.

[8](美)哈伯德（Hubbard,E）·你属于哪种人[M]·北京：机械上出版社，2003.

[9] 顾械刚·名家谈孟姜女哭长城[M]·北京：文化艺术出版社，2006.

[10] 朱大可、张阁·21 世纪中国文化地图[M]·桂林: 广西师范大 学出版社, 2005.

[11] 李欣·长城，世界一大奇迹/成大林著-上海[M]: 上海文艺术出版社，2006.

[12] 黄瑞旗·孟姜女故事研究[M]·桂林：中国人民大学出版社， 2003.

[13] 顾希佳·孟姜女哭长城[M]·广东: 广东教育出版社, 2006.

[14] 林可心·孟姜女与范喜良 [M]·福建：福建教育出版社， 2007.

[15] 洪淑苓·孟姜女故事形塑的女性文化及其在歌仔册文本的呈现[J]·东海中文学报, 2008,(20):55-88.

[16] 柏生·气质[Z]·http://baike.baidu.com/ view/5309.htm?fr=ala0_1_1 2010-04-23/2010-05-01.

[17] 苏童[Z]·http://baike.baidu.com/view/89168.htm?fr=ala0_1_1·2010-02-19/2010-03-19.

[18] 孟姜女[Z]·http://baike.baidu.com/view/14001.htm?fr=ala0_1_1·2010-03-23/2010-0408.

[19] Kinmark·孟姜女庙[Z]·http://baike.baidu.com/ view/16116.htm?fr=ala0_1_1·2010-5-20/2010-6-8.

[20] Briskly·性格[z]·http://baike.baidu.com/view/50208.htm?fr=ala0_1_1·2010-05-02/2010-05-05.

[21]刘昱 中国神话：孟姜女性格分析[Z] http://www.univs.cn/newweb/univs/hust/2006-11-14/ 697900.html.2006-11-14/2009-02-25.

\section{印尼文文献}

[1] Gea, A. A., Wulandari, A. P. Y., dan Babari, Y. (2002). Modul Character dengan Diri Sendiri. Jakarta: Elex Media Komputindo.

Building: Relasi

[2] Fred, dan Litataur, F. (2003). Why Do I Fell The Way I Do ?(Mengapakah Aku Merasa Seperti Ini?). Jakarta: Binarupa Aksara.

[3] Littauer, F. (1997). Pohon Kepribadian Anda. Jakarta: Binarupa Aksara.

[4]Littauer, F., dan Littauer, M. (1997). Personality Puzzle (Teka-teki Kepribadian). Jakarta: Professional Books.

[5] Littauer, F. (1998). Personality Plus. Jakarta: Binarupa Aksara. 
[6] Fitri, A.Sanguinis, Melankolis, Koleris atau Plegmatis ya?’.Multiply.24 May 2007.13 Mar 2010 $<$ http://sahabatjiwa.multiply.com/journal/item/6/Sanguinis_Melankolis_Koleris_ atau_Plegmatis_ya>.

[7] Mase, S. Karakter Manusia Versi Hipocrates. My Opera . 19 Jun 2009.15 Mar $2010<$ http: //my.opera.com/Mysukma/blog/karakter-manusia-versi-hipocrates? startidx $=450>$. 